\title{
Multidimensional Risk Factors of Age-Related Hearing Loss Among Malaysian Community- Dwelling Older Adults
}

\author{
Theng Choon Ooi ${ }^{\prime}$ \\ Wan Syafira Ishak (D) \\ Razinah Sharif' \\ Suzana Shahar' \\ Nor Fadilah Rajab' \\ Devinder Kaur Ajit Singh (D) ' \\ Siti Zamratol-Mai Sarah Mukari ${ }^{2}$ \\ 'Centre for Healthy Ageing and Wellness, \\ Faculty of Health Sciences, Universiti \\ Kebangsaan Malaysia, Kuala Lumpur, \\ 50300, Malaysia; ${ }^{2}$ Institute of Ear, Hearing \\ and Speech, Universiti Kebangsaan \\ Malaysia, Kuala Lumpur, 50300, Malaysia
}

Correspondence: Wan Syafira Ishak Audiology Program, Centre for Healthy Ageing and Wellness, Faculty of Health Sciences, Universiti Kebangsaan Malaysia, Jalan Raja Muda Abdul Aziz, Kuala Lumpur, 50300, Malaysia

Tel +603928950 II

Fax +60392897161

Email wsyafira@ukm.edu.my
Purpose: This study evaluates the prevalence of and the multidimensional risk factors associated with age-related hearing loss (ARHL) among community-dwelling older adults in Malaysia.

Patients and Methods: A total of 253 participants aged 60 years and above participated in this cross-sectional study. The participants were subjected to pure tone audiometric assessment. The hearing threshold was calculated for the better ear and classified into pure-tone average (PTA) for the octave frequencies from 0.5 to $4 \mathrm{kHz}$ and high-frequency pure-tone average (HFA) for the octave from 2 to $8 \mathrm{kHz}$. Then, the risk factors associated with PTA hearing loss (HL) and HFAHL were identified by using multivariate logistic regression analysis.

Results: The prevalence of ARHL based on PTA and HFA among the community-dwelling older adults was $75.5 \%$ and $83.0 \%$, respectively. Following multifactorial adjustments, being older (OR: 1.239; 95\% CI: 1.062-1.445), having higher waist circumference (OR: 1.158; 95\% CI: 1.015-1.322), lower intake of niacin (OR: 0.909; 95\% CI: 0.831-0.988) and potassium (OR: 0.998; 95\% CI: 0.996-1.000), and scoring lower in RAVLT T5 (OR: 0.905; 95\% CI: 0.838-0.978) were identified as the risk factors of PTAHL. Meanwhile, being older (OR: 1.117; 95\% CI: 1.003-1.244), higher intake of carbohydrate (OR: 1.018; 95\% CI: 1.006-1.030), lower intake of potassium (OR: 0.998; 95\% CI: 0.997-0.999), and lower scores on the RAVLT T5 (OR: 0.922; 95\% CI: 0.874-0.973) were associated with increased risk of having HFAHL.

Conclusion: Increasing age, having higher waist circumference, lower intake of niacin and potassium, higher intake of carbohydrates and having lower RAVLT T5 score were associated with increased risk of ARHL. Modifying these risk factors may be beneficial in preventive and management strategies of ARHL among older persons.

Keywords: cognitive function, dietary intake, hearing loss, older adults, prevalence, risk factors

\section{Introduction}

Age-related hearing loss (ARHL) is an age-related degenerative disease characterized by progressive symmetrical sensorineural hearing loss (HL) predominantly at higher frequencies. ${ }^{1}$ Approximately 1.5 billion people experience a certain degree of HL worldwide, with more than $42 \%$ of them being older adults aged 60 years and above. ${ }^{2}$ It is reported that over $65 \%$ of individuals older than 60 years old have HL, and the prevalence increases exponentially with age among the older populations. ${ }^{3}$ The prevalence of ARHL is expected to increase dramatically due 
to the increasing aging population worldwide, as observed in the current trend of the global population shift. ${ }^{2}$

The underlying causes of ARHL are complex and multifactorial. Its development and progression could be attributed to predisposing genetic and biological risk factors, comorbidities, lifestyle behaviors and environmental insults. ${ }^{1,4}$ Predisposing genetic factors play a crucial role in determining the onset and severity of ARHL. Meanwhile, men are more likely to develop ARHL than women of the same age, probably due to a higher risk of exposure to noise or other environmental risk factors. ${ }^{2}$ Individuals with certain health conditions such as cardiovascular diseases, diabetes and renal diseases are at greater risk of ARHL. ${ }^{4}$ The use of ototoxic drugs, constant exposure to occupational hazards such as noise and chemicals, as well as practicing unhealthy lifestyle habits (eg, smoking, excessive intake of alcohol, unhealthy eating habits, nutritional deficiency and exposure to excessive loudness in recreational settings) appeared as the common risk factors of such sensory deficit. ${ }^{1,4}$ All these risk factors can initiate and exacerbate the progression of ARHL independently or synergistically.

ARHL is the third largest cause of years lived with disability (YLDs) in 2019 and the leading cause of YLDs for older adults over 70 years. ${ }^{3}$ Due to its high prevalence globally, ARHL posts significant economic impacts on society. ${ }^{5}$ Moreover, unaddressed HL among older adults may lead to cognitive, physical and psychosocial problems such as social isolation, functional loss, depression, cognitive impairment and physical frailty. ${ }^{1,4}$ Early screening for ARHL and the use of hearing aids have been reported to mitigate the adverse effects and improve the quality of life of those affected. However, the effectiveness of such interventional approaches is limited by several factors, including lack of accessibility to related healthcare facilities providing specialized hearing care, stigma and inability to afford hearing aids. ${ }^{5}$ Identification of risk factors, especially modifiable risk factors (eg, lifestyle habits and environmental insults), may help plan and develop preventive strategies to reduce the progression and severity of ARHL. However, study focus on ARHL is limited in Malaysia. The National Ear and Hearing Disorders Survey completed in 2005 found that $69.9 \%$ of older adults aged 60 years and above have HL. ${ }^{6}$ While the study reported on demographic trends of HL, it did not provide a detailed investigation of modifiable risk factors of HL. Hence, the main objective of this study was to determine the multidimensional risk factors of ARHL among community-dwelling older adults in Malaysia.

\section{Materials and Methods Study Cohort}

This study was part of a prospective population-based study on aging [Long-term Research Grant Scheme Towards Useful Ageing (LRGS-TUA)]. The data were collected from the first wave of the LRGS-TUA study conducted in the year 2013. The older adults aged 60 and above were recruited through a stratified random sampling method from four different states in Malaysia, which were Selangor (central part of Malaysia), Perak (north-west), Kelantan (north-east) and Johor (southern part). The audiometric assessment data, however, was only available for participants from Selangor. A total of 573 older adults fulfilled the inclusion criteria and agreed to participate. However, only 253 participants (44.2\%) completed all the testing were included in this study. The sampling method, inclusion criteria and exclusion criteria for this study were described in detail by Shahar et al. $^{7}$

\section{Ethics Approval and Informed Consent}

This study was approved by the Medical Research and Ethics Committee of Universiti Kebangsaan Malaysia (UKM 1.5.3.5/244/NN-060-2013) and was conducted in accordance with the Declaration of Helsinki. Written informed consent was obtained from the participants before their participation.

\section{Data Collection}

Data on socio-demographic, medical history, dietary status, cognitive function, psychosocial and functional status of the participants were obtained by trained field workers through face-to-face interviews using standardized questionnaires. ${ }^{7}$ The data on anthropometric, body composition, blood pressure, physical fitness, biochemical and audiometric were assessed using protocols as described by Shahar et al. ${ }^{7}$

\section{Socio-Demographic Data and Medical History}

The information obtained included the age, sex, ethnicity, smoking and alcohol drinking habits, years of education and self-reported medical history (hypertension, hypercholesterolemia, diabetes mellitus, cardiovascular disease and tinnitus). 


\section{Anthropometric, Body Composition and Blood Pressure Measurements}

The body height and weight were measured using a portable SECA 206 portable body meter (Seca, Hamburg, Germany) and Tanita digital lithium weighing scale (Tanita, Tokyo, Japan). Then, the body mass index (BMI) was computed by using the formula "body weight $(\mathrm{kg})$ /height $(\mathrm{m})^{2}$ ". The waist, hip and calf circumferences were measured using Lufkin tape, and the readings were taken to the nearest $0.1 \mathrm{~cm}$. Body composition (fat mass, fat-free mass, skeletal muscle mass and body fat percentage) was analyzed using a Bioelectrical Impedance Analysis Inbody S10 (Biospace, Seoul, Korea). The systolic and diastolic blood pressure was measured twice consecutively using an automatic digital blood pressure monitor (OMRON, Kyoto, Japan) to get the average reading.

\section{Assessment of Dietary Intake}

Participants' usual food and drinks intake in a week were recorded using a validated Dietary Habits Questionnaire specialized for older adults. ${ }^{7}$ Then, the dietary record of each participant was analyzed by using the Nutritionist $\mathrm{Pro}^{\mathrm{TM}}$ Software to obtain their respective nutrient intake profile.

\section{Blood Sample Collection and Biochemical Analysis} Participants were instructed to fast overnight before blood sample collection. The fasting venous blood was then collected from the participants by a trained phlebotomist and was sent to accredited medical laboratories for biochemical analysis. The parameters included in the analysis are fasting blood glucose, hemoglobin, total cholesterol, high-density lipoprotein (HDL), low-density lipoprotein (LDL), triglyceride and albumin levels.

\section{Physical Fitness Assessment}

The participants' physical fitness was assessed using the Senior Fitness Test. ${ }^{7}$ The Senior Fitness Test is a battery of tests, including a 2-minute step test, chair stand test, chair sit-and-reach test, timed up-and-go test, dominant handgrip strength test and back scratch test. These fitness tests were used to measure aerobic endurance, lower limb muscle strength, lower body flexibility, mobility and balance, upper limb muscle strength and upper body flexibility.

\section{Cognitive Function Assessment}

The Malay version of Mini-Mental State Examination (MMSE) and Montreal Cognitive Assessment (MoCA) were used to evaluate global function. Despite their similarity in cognitive function evaluation, the MoCA test is of greater sensitivity to detect mild cognitive impairment with less ceiling effect than MMSE. ${ }^{8}$ Meanwhile, the Digit Symbol Test and Digit Span Test, the subsets of the Wechsler Adult Intelligence Scale, were used to evaluate the information processing, visual-motor speed, visual memory and coordination and attention, concentration and memory, respectively. Then, the Rey Auditory Verbal Learning Test (RAVLT) was used to determine short-term verbal memory and verbal learning. The cognitive function assessment was described in detail by Shahar et al. ${ }^{7}$

\section{Psychosocial and Functional Status Assessment}

Activities of Daily Living (ADL), Instrumental Activity of Daily Living (IADL) and World Health Organization Disability Assessment Schedule 2.0 (WHODAS 2.0) were used to assess the functioning in self-care, independent living skills and disability, respectively. Meanwhile, the Medical Outcome Social Support (MOSS) survey was used to measure functional social support. Personality disorder and depressive symptoms were determined by using Eysenck Personality Questionnaire and Geriatric Depression Scale. The feelings of loneliness, perception of stress, self-perceived success, and life satisfaction were evaluated using the three-item Loneliness Scale, four-item Perceived Stress Scale, eight-item Flourishing Scale and Satisfaction with Life Scale, respectively. The psychosocial and functional status assessment was described in detail by Shahar et al. ${ }^{7}$

\section{Audiometric Assessment}

The audiometric assessment was carried out with slight modification from Mukari et al method. ${ }^{9}$ Briefly, the hearing status of the participants was carried out by trained personnel in a sound-treated mobile booth using a calibrated Madsen Itera II diagnostic audiometer with TDH 39 headphones. The air-conduction thresholds for left and right ears were obtained monaurally at frequencies of $0.5,1,2,3,4,6$ and $8 \mathrm{kHz}$. In this study, we categorized the hearing thresholds in the better ear as pure-tone average (PTA) for the octave frequencies from 0.5 to $4 \mathrm{kHz}$ and high-frequency pure-tone average (HFA) for the octave from 2 to $8 \mathrm{kHz}$. The HL is defined as a threshold average greater than $25 \mathrm{~dB}$ hearing level.

\section{Statistical Analysis}

The data were analyzed using the Statistical Package for the Social Sciences version 25.0 (IBM Corp, Armonk, New York, USA). The prevalence of HL is calculated by 
dividing the number of participants with HL based on PTA (PTAHL) or HFA (HFAHL) by the total number of participants in this study. Next, the variables were compared between participants with normal hearing and HL of each PTA category by using independent $T$-test for continuous variables or Chi-square test for categorical variables. The variables that appeared to be significant $(p<0.05)$ in the univariate tests were further subjected to binary logistic regression analysis. Then, variables with significant $(\mathrm{p}<$ 0.05 ) associations with HL were included in the final multivariate binary logistic regression model and were adjusted with other well-known confounding factors (age, sex, ethnicity, years of education, smoking and alcohol drinking habits, multimorbidity and BMI). Variables that were found to be significant $(p<0.05)$ in the final multivariate logistic regression model were identified as the potential risk factors of PTAHL or HFAHL.

\section{Results}

The characteristics of the participants included and excluded from the analysis are presented in Table 1. There are no significant differences in most of the characteristics, except for the proportion of ethnic $(\mathrm{p}<0.01)$, with higher percentage of Malay population and lower Chinese and Indian populations among the excluded participants.

The hearing loss based on HFA among communitydwelling older adults was more prevalent (83\%) than hearing loss based on the PTA criterion (75.5\%). Table 2 shows the characteristics of the participants based on their hearing status. Compared to participants with normal hearing, participants with PTAHL were more likely $(\mathrm{p}<0.05)$ to be men $(47.6 \%)$, older $(69.49 \pm 6.07$ years $)$, Chinese (59.7\%), have cardiovascular diseases (16.8\%), higher waist circumference $(91.24 \pm 11.93 \mathrm{~cm})$, higher body fat mass $(25.21 \pm 8.89 \mathrm{~kg})$, lower intake of vitamin $\mathrm{C}(124.83$ $\pm 69.11 \mathrm{mg} /$ day $)$, niacin $(9.55 \pm 3.50 \mathrm{mg} /$ day $)$, potassium $(1355.39 \pm 425.50 \mathrm{mg} /$ day $)$, zinc $(3.30 \pm 1.45 \mathrm{mg} /$ day $)$ and copper $(0.61 \pm 0.27 \mathrm{mg} /$ day $)$, higher fasting blood glucose level $(6.44 \pm 2.27 \mathrm{mmol} / \mathrm{L})$, had lower chair sit-and-reach test $(9.61 \pm 12.39 \mathrm{~cm})$, timed up and go test $(10.44 \pm 3.21$ seconds) and back scratch test $(19.05 \pm 13.98 \mathrm{~cm})$ scores, scored lower on the MMSE (23.47 \pm 4.94$)$, MoCA (19.39 $\pm 6.12)$, Digit Symbol Test $(5.91 \pm 3.05)$, Digit Span Test $(7.67 \pm 2.68)$ and RAVLT T5 score $(37.97 \pm 11.41)$, had higher WHODAS $2.0(3.90 \pm 6.30)$ and flourishing scale $(14.89 \pm 7.26)$ scores.
Meanwhile, the participants with HFAHL were more likely to be men (48.1\%), older ( $69.30 \pm 5.99$ years), have cardiovascular diseases (15.7\%), higher waist circumference $(90.94 \pm 11.91 \mathrm{~cm})$, higher intake of carbohydrate (220.31 $\pm 62.05 \mathrm{~g} /$ day), lower intake of vitamin C (126.29 $\pm 69.42 \mathrm{mg} /$ day $)$, potassium (1376.04 $\pm 453.15 \mathrm{mg} /$ day $)$, magnesium (135.51 $\pm 54.32 \mathrm{mg} /$ day), zinc $(3.36 \pm$ $1.46 \mathrm{mg} /$ day $)$ and copper $(0.62 \pm 0.31 \mathrm{mg} /$ day $)$, higher fasting blood glucose level $(6.38 \pm 2.20 \mathrm{mmol} / \mathrm{L})$, lower HDL level $(1.40 \pm 0.35 \mathrm{mmol} / \mathrm{L})$, had lower chair sit-andreach test $(9.43 \pm 12.22 \mathrm{~cm})$, timed up and go test $(10.29 \pm$ 3.15 seconds) and back scratch test $(18.92 \pm 13.99 \mathrm{~cm})$ scores, scored lower in MMSE (23.65 \pm 4.86$)$, MoCA $(19.63 \pm 6.04)$, Digit Symbol Test $(6.06 \pm 3.06)$, Digit Span Test $(7.71 \pm 2.68)$ and RAVLT T5 score (38.15 \pm 11.33) and scored higher in WHODAS 2.0 (3.60 \pm 6.11$)$.

The variables that are significantly associated with the PTAHL and HFAHL as analyzed using the binary logistic regression are listed in Table 3. These variables were then entered into the final multiple logistic regression model to determine the risk factors associated with both types of HL (Table 4). Following multifactorial adjustments, being older (OR: 1.239; 95\% CI: 1.062-1.445), having higher waist circumference (OR: 1.158; 95\% CI: 1.015-1.322), lower intake of niacin (OR: 0.909; 95\% CI: 0.831-0.988) and potassium (OR: 0.998; 95\% CI: 0.996-1.000), and scored lower in RAVLT T5 (OR: 0.905; 95\% CI: 0.8380.978 ) were identified as the risk factors of PTAHL. In the meantime, increasing age (OR: 1.117; 95\% CI: 1.0031.244), higher intake of carbohydrate (OR: 1.018; 95\% CI: 1.006-1.030), lower intake of potassium (OR: 0.998; 95\% CI: 0.997-0.999), and scored lower in RAVLT T5 (OR: 0.922; 95\% CI: 0.874-0.973) were associated with increased risk of having HFAHL. Being older, lower intake of potassium and lower RAVLT T5 score appeared to be the common risk factors for PTAHL and HFAHL.

\section{Discussion}

We found that the prevalence of PTAHL among Malaysian older adults aged 60 years and above was 75.5\%. This prevalence is within the range of $69.9-76.2 \%$, as reported in previous studies. ${ }^{6,10,11}$ However, the National Health Morbidity Survey (NHMS) 2018 showed that the prevalence of self-reported hearing disability in older Malaysians aged 60 years and above was only $6.4 \%{ }^{12}$ The marked differences in the prevalence rate between our current findings and the NHMS 2018 could be attributed to different approaches to detect hearing impairment. 
Table I The Characteristics of the Included and Excluded Participants in the Study

\begin{tabular}{|c|c|c|c|c|}
\hline \multirow[t]{2}{*}{ Characteristic } & \multicolumn{4}{|c|}{ n (\%) or Mean \pm SD } \\
\hline & $\begin{array}{l}\text { Total Eligible Participants } 573 \\
\qquad(100)\end{array}$ & $\begin{array}{c}\text { Included Participants } 253 \\
\text { (44.2) }\end{array}$ & $\begin{array}{c}\text { Excluded Participants } 320 \\
\text { (55.8) }\end{array}$ & p-value \\
\hline Age & $68.30 \pm 6.07$ & $68.59 \pm 5.92$ & $68.08 \pm 6.19$ & 0.318 \\
\hline \multicolumn{5}{|l|}{ Sex } \\
\hline Male & $243(42.4)$ & $109(43.1)$ & I34 (4I.9) & 0.771 \\
\hline Female & $330(57.6)$ & I 44 (56.9) & $186(58.1)$ & \\
\hline \multicolumn{5}{|l|}{ Ethnicity } \\
\hline Malay & $|8|(3 \mid .6)$ & $57(22.5)$ & $124(38.8)$ & $0.001 * *$ \\
\hline Chinese & $291(50.8)$ & $144(56.9)$ & 147 (45.9) & \\
\hline Indian & $96(16.8)$ & $50(19.8)$ & $46(14.4)$ & \\
\hline Others & $5(0.9)$ & $2(0.8)$ & $3(0.9)$ & \\
\hline \multicolumn{5}{|l|}{ Smoking } \\
\hline Yes & $47(8.2)$ & $21(8.3)$ & $26(8.1)$ & 0.939 \\
\hline No & $526(91.8)$ & $232(91.7)$ & $294(91.9)$ & \\
\hline \multicolumn{5}{|l|}{ Drinking alcohol } \\
\hline Yes & $47(8.2)$ & $27(10.7)$ & $20(6.3)$ & 0.055 \\
\hline No & $526(91.8)$ & $226(89.3)$ & $300(93.8)$ & \\
\hline Education (years) & $6.86 \pm 4.70$ & $6.55 \pm 4.66$ & $7.11 \pm 4.72$ & 0.156 \\
\hline \multicolumn{5}{|l|}{ Medical history } \\
\hline Hypertension & $328(57.2)$ & $140(55.3)$ & $188(58.8)$ & 0.412 \\
\hline Hypercholesterolemia & $240(41.9)$ & $110(43.5)$ & $130(40.6)$ & 0.492 \\
\hline Cardiovascular disease & 67 (II.7) & $34(13.4)$ & $33(10.3)$ & 0.248 \\
\hline Diabetes mellitus & $180(31.4)$ & $82(32.4)$ & $98(30.6)$ & 0.647 \\
\hline \multicolumn{5}{|l|}{ Physical measurement } \\
\hline BMI $\left(\mathrm{kg} / \mathrm{m}^{2}\right)$ & $25.70 \pm 4.36$ & $25.31 \pm 4.23$ & $26.01 \pm 4.44$ & 0.057 \\
\hline $\begin{array}{l}\text { Waist circumference } \\
(\mathrm{cm})\end{array}$ & $90.43 \pm 11.74$ & $90.05 \pm 11.96$ & $90.73 \pm 11.56$ & 0.491 \\
\hline Hip circumference $(\mathrm{cm})$ & $99.59 \pm 9.17$ & $99.03 \pm 8.98$ & $100.03 \pm 9.32$ & 0.195 \\
\hline Calf circumference $(\mathrm{cm})$ & $34.78 \pm 3.70$ & $34.67 \pm 3.37$ & $34.86 \pm 3.95$ & $0.54 I$ \\
\hline Systolic (mmHg) & $137.12 \pm 19.32$ & $135.88 \pm 19.01$ & $138.1 \mid \pm 19.54$ & 0.180 \\
\hline Diastolic (mmHg) & $76.54 \pm 12.57$ & $76.52 \pm 12.44$ & $76.55 \pm 12.70$ & 0.977 \\
\hline
\end{tabular}

Note: $* * p<0.01$.

Abbreviation: BMI, body mass index.

This present study used pure tone audiometry to assess the hearing status of participants, which is the gold standard test for hearing acuity. ${ }^{2}$ Meanwhile, the NHMS 2018 used the self-reporting Washington Group of Disability questionnaires to evaluate hearing disability among older adults. ${ }^{12}$ Hence, variations may occur since the outcome measurements of these two assessments are of two different perspectives. Although the use of the selfreported hearing questionnaire is of notable advantages over the PTA assessment in terms of cost and ease of administration, biases may occur due to the interindividual variability of perception and belief on hearing impairments, resulting in underestimation or overestimation of HL. ${ }^{13,14}$ The accuracy of self-reported HL is also 


\begin{tabular}{|c|c|c|c|c|c|c|c|c|c|c|c|c|c|c|c|}
\hline & 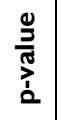 & 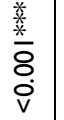 & & 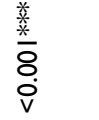 & & $\begin{array}{l}\stackrel{\text { }}{\hat{R}} \\
\text { o }\end{array}$ & & $\begin{array}{l}\bar{f} \\
\text { o. }\end{array}$ & & $\begin{array}{l}\frac{j}{4} \\
0\end{array}$ & 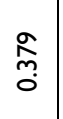 & & 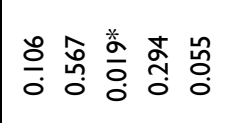 & & 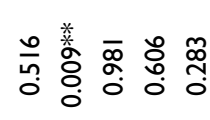 \\
\hline & 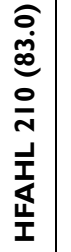 & $\begin{array}{l}2 \\
\sigma \\
i \\
+1 \\
0 \\
0 \\
0 \\
0\end{array}$ & & 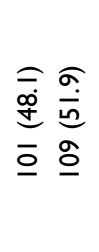 & & 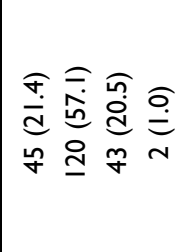 & & 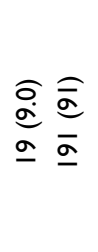 & & 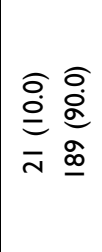 & $\begin{array}{l}\hat{n} \\
+ \\
+1 \\
+1 \\
\tilde{q} \\
0\end{array}$ & & 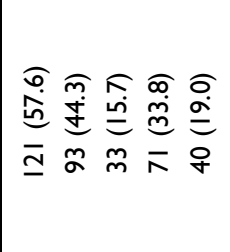 & & 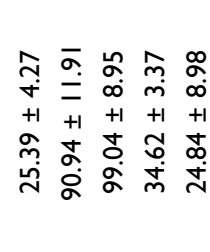 \\
\hline & 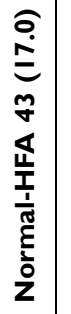 & 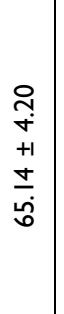 & & 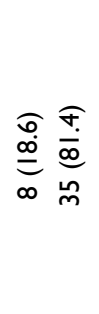 & & 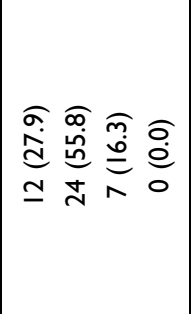 & & 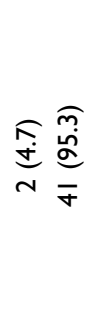 & & 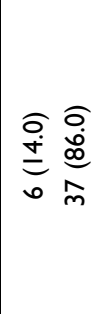 & $\begin{array}{l}0 \\
0 \\
i \\
+1 \\
+1 \\
\end{array}$ & & 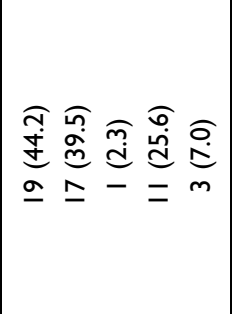 & & 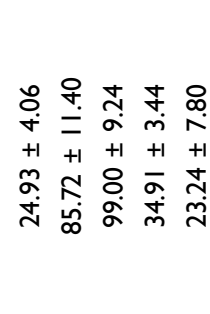 \\
\hline $\begin{array}{l}\text { Q } \\
+1 \\
\text { ह } \\
\Sigma \\
\Sigma\end{array}$ & 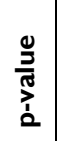 & 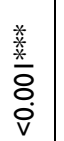 & & $\frac{*}{0}$ & & 誊 & & ڤ્તి & & $\begin{array}{l}\stackrel{\circ}{0} \\
\infty \\
0\end{array}$ & $\begin{array}{l}\text { ڤo } \\
\stackrel{0}{0}\end{array}$ & & 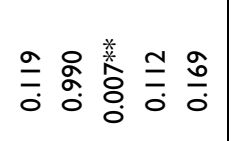 & & 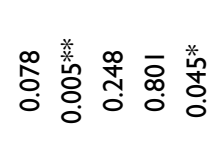 \\
\hline$\underset{\mathbf{c}}{\stackrel{0}{0}}$ & 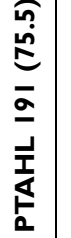 & $\begin{array}{l}\hat{0} \\
\dot{0} \\
+1 \\
o \\
\sigma \\
0 \\
0\end{array}$ & & 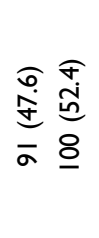 & & 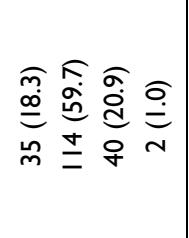 & & 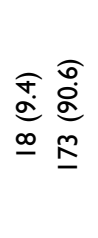 & & 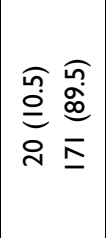 & 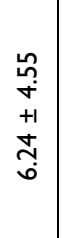 & & 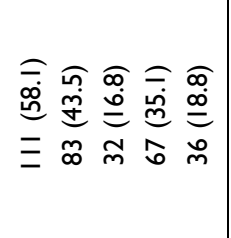 & & 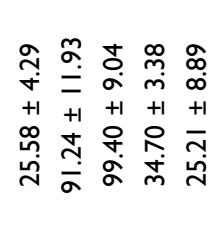 \\
\hline & 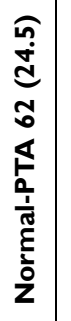 & 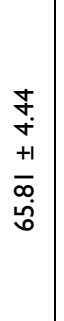 & & 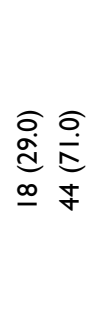 & & 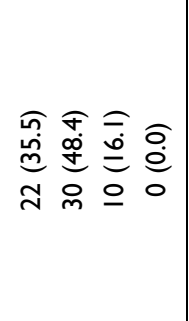 & & 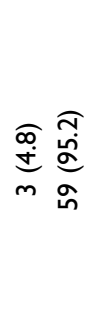 & & 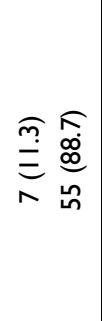 & $\begin{array}{l}2 \\
\stackrel{+}{+} \\
+1 \\
\circ \\
\stackrel{n}{n}\end{array}$ & & 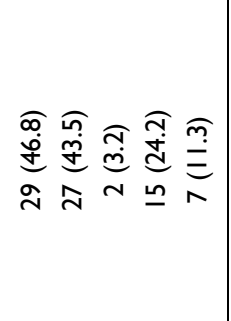 & & 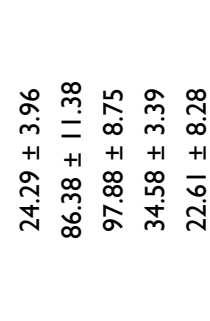 \\
\hline & 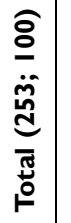 & 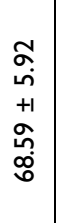 & & 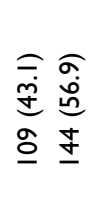 & & 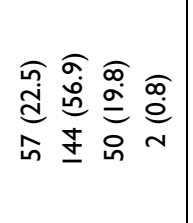 & . & 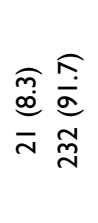 & & 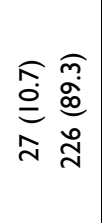 & 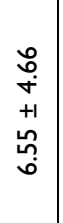 & & 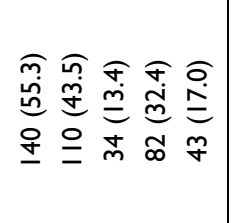 & & 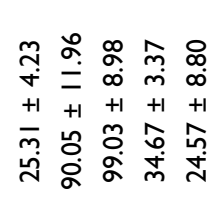 \\
\hline 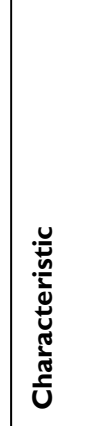 & & $\stackrel{8}{80}$ & ڤ્㐅 & 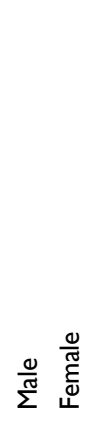 & 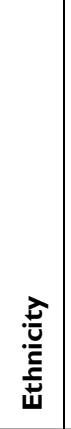 & 离 & 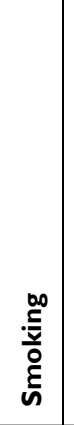 & 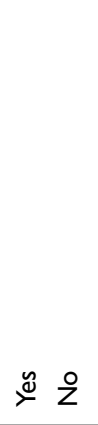 & 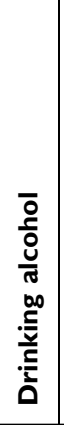 & $\underbrace{\stackrel{y}{u}}_{\sim}$ z & 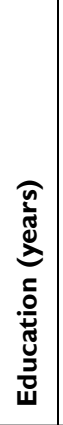 & 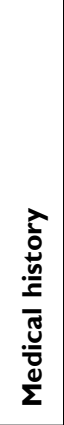 & 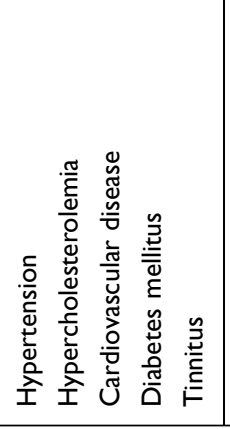 & 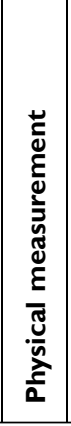 & 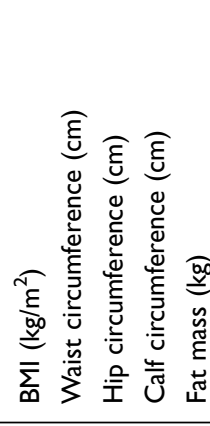 \\
\hline
\end{tabular}




\begin{tabular}{|c|c|c|c|c|}
\hline 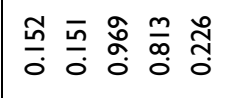 & & 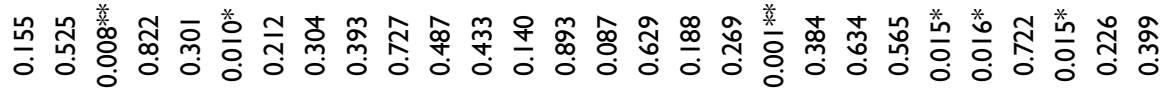 & & $\stackrel{\frac{*}{0}}{\overline{0}}$ \\
\hline 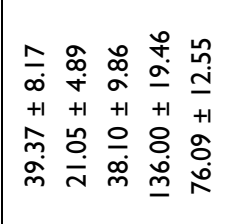 & & 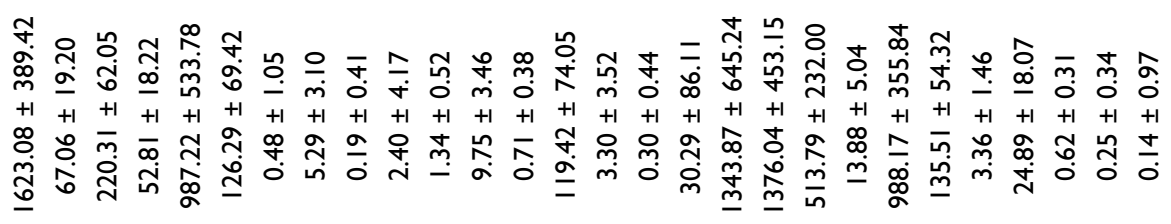 & & 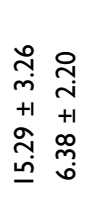 \\
\hline 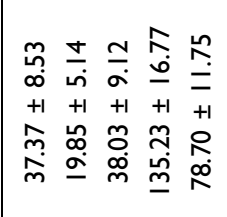 & & 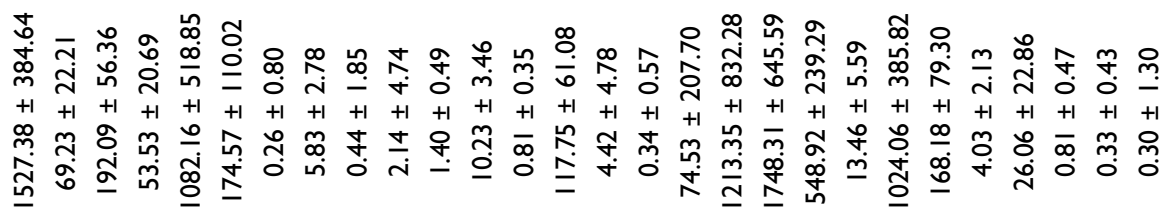 & & 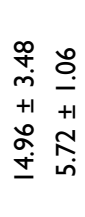 \\
\hline 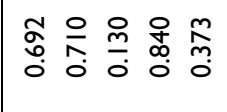 & & 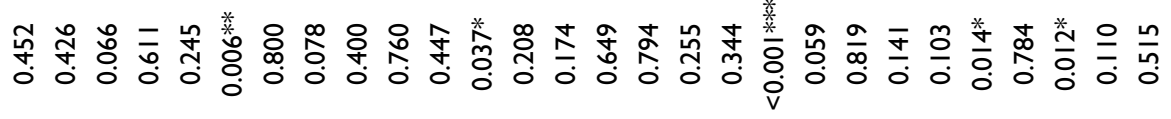 & & 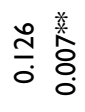 \\
\hline 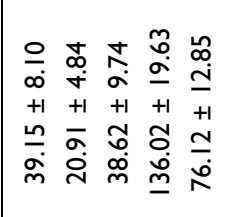 & & 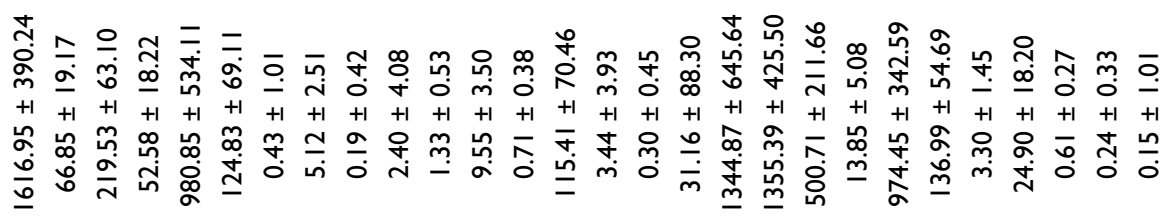 & & 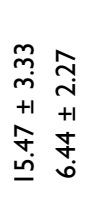 \\
\hline 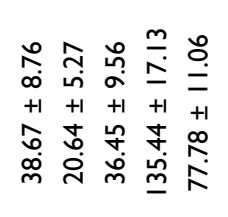 & & 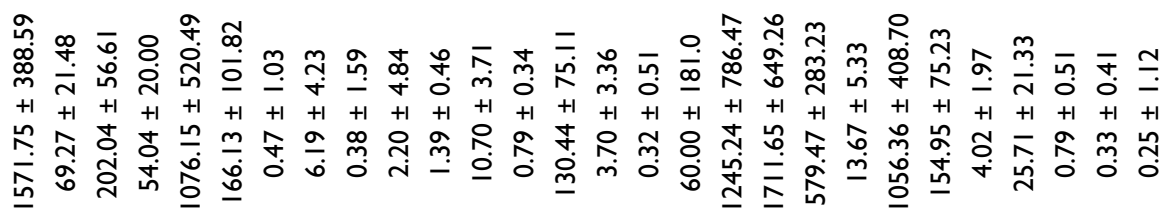 & & 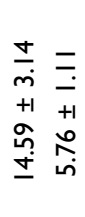 \\
\hline 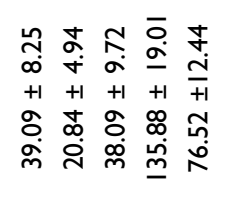 & & 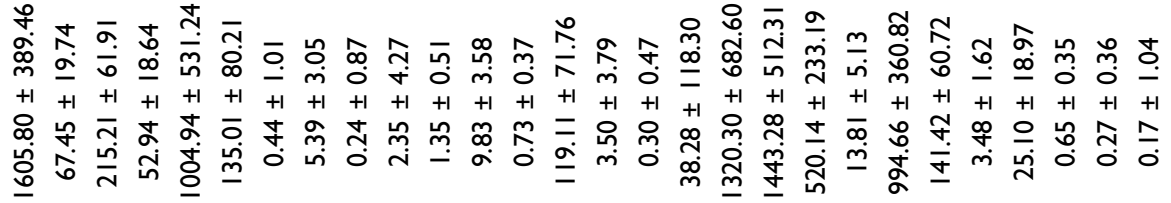 & & 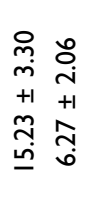 \\
\hline 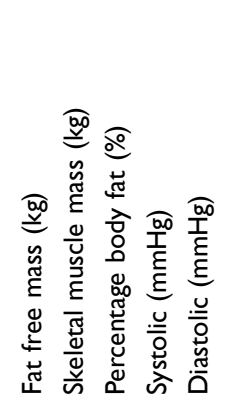 & 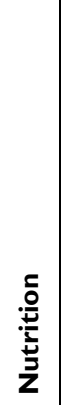 & 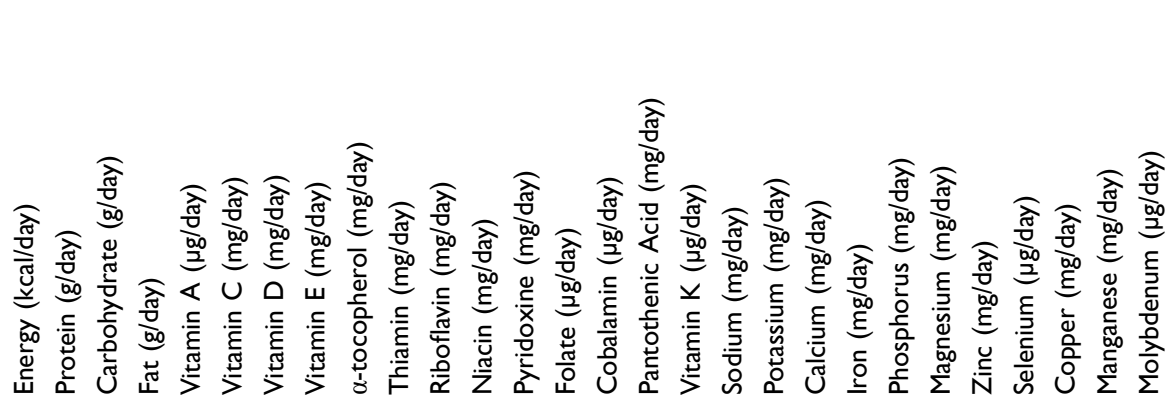 & 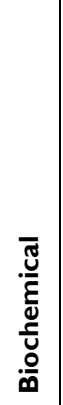 & 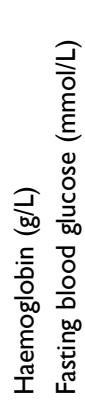 \\
\hline
\end{tabular}




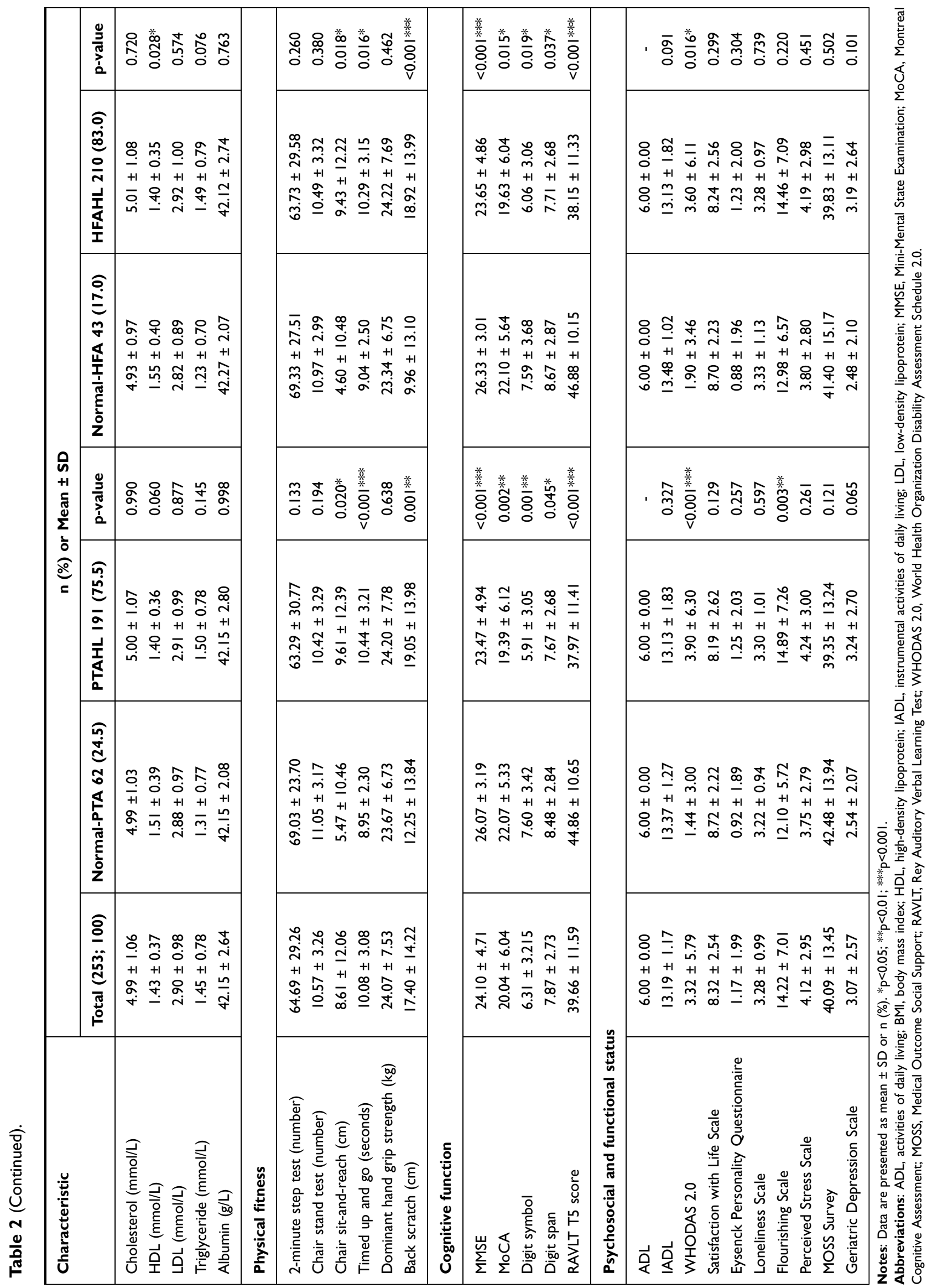


Table 3 The Univariate Scores for Individual Risk Factors of PTAHL and HFAHL

\begin{tabular}{|c|c|c|c|c|c|c|c|}
\hline \multirow[t]{2}{*}{ Risk Factors Category } & \multirow[t]{2}{*}{ Item } & \multicolumn{3}{|c|}{ PTAHL } & \multicolumn{3}{|c|}{ HFAHL } \\
\hline & & p-value & $\operatorname{Exp}(B)$ & {$[95 \% \mathrm{Cl}]$} & p-value & $\operatorname{Exp}(B)$ & {$[95 \% \mathrm{Cl}]$} \\
\hline Sociodemographic & $\begin{array}{l}\text { Age } \\
\text { Sex (Male) } \\
\text { - Female } \\
\text { - Male } \\
\text { Ethnicity } \\
\text { - Malay } \\
\text { - Chinese } \\
\text { - Indian } \\
\text { - Others }\end{array}$ & $\begin{array}{c}<0.00 I^{* * *} \\
\text { Reference } \\
0.011^{*} \\
\text { Reference } \\
0.01 I^{*} \\
0.039 * \\
0.999\end{array}$ & $\begin{array}{c}1.135 \\
\text { Reference } \\
2.224 \\
\text { Reference } \\
2.389 \\
2.514 \\
-\end{array}$ & $\begin{array}{c}\text { I.068-1.206 } \\
\text { Reference } \\
\text { I.200-4.125 } \\
\text { Reference } \\
\text { I.225-4.658 } \\
\text { I.049-6.028 } \\
-\end{array}$ & $\begin{array}{l}<0.00 I^{* * *} \\
\text { Reference } \\
0.00 I^{* *} \\
- \\
- \\
- \\
-\end{array}$ & $\begin{array}{c}1.166 \\
\text { Reference } \\
4.054 \\
- \\
- \\
- \\
-\end{array}$ & $\begin{array}{c}\text { I.08I-I.258 } \\
\text { Reference } \\
\text { I.796-9.153 } \\
- \\
- \\
- \\
-\end{array}$ \\
\hline Medical history & $\begin{array}{l}\text { Cardiovascular diseases } \\
\text { - No } \\
\text { - Yes }\end{array}$ & $\begin{array}{c}\text { Reference } \\
0.016^{*}\end{array}$ & $\begin{array}{c}\text { Reference } \\
6.038\end{array}$ & $\begin{array}{c}\text { Reference } \\
\text { I.403-25.975 }\end{array}$ & $\begin{array}{c}\text { Reference } \\
\text { 0.046* }\end{array}$ & $\begin{array}{c}\text { Reference } \\
7.831\end{array}$ & $\begin{array}{c}\text { Reference } \\
\text { I.04I-58.892 }\end{array}$ \\
\hline Physical measurement & $\begin{array}{l}\text { Waist circumference } \\
\text { Fat mass }\end{array}$ & $\begin{array}{l}0.006 * * \\
0.047 *\end{array}$ & $\begin{array}{l}1.036 \\
1.036\end{array}$ & $\begin{array}{l}1.010-1.063 \\
1.001-1.073\end{array}$ & $\begin{array}{c}0.010^{*} \\
-\end{array}$ & $\begin{array}{c}1.039 \\
-\end{array}$ & $\begin{array}{c}1.009-1.070 \\
-\end{array}$ \\
\hline Nutrition & $\begin{array}{l}\text { Carbohydrate } \\
\text { Vitamin C } \\
\text { Niacin } \\
\text { Potassium } \\
\text { Magnesium } \\
\text { Zinc } \\
\text { Copper }\end{array}$ & $\begin{array}{c}- \\
0.00 I^{* *} \\
0.040^{*} \\
<0.00 I^{* * *} \\
- \\
0.006^{* *} \\
0.002^{* *}\end{array}$ & $\begin{array}{c}- \\
0.994 \\
0.917 \\
0.999 \\
- \\
0.773 \\
0.252\end{array}$ & $\begin{array}{c}- \\
0.990-0.998 \\
0.845-0.996 \\
0.998-0.999 \\
- \\
0.642-0.930 \\
0.105-0.603\end{array}$ & $\begin{array}{c}0.009 * * \\
0.00 I^{* *} \\
- \\
<0.00 I^{* * *} \\
0.004^{* *} \\
0.02 I^{*} \\
0.004^{* *}\end{array}$ & $\begin{array}{c}1.009 \\
0.993 \\
- \\
0.999 \\
0.992 \\
0.797 \\
0.267\end{array}$ & $\begin{array}{c}1.002-1.015 \\
0.990-0.997 \\
- \\
0.998-0.999 \\
0.987-0.997 \\
0.657-0.966 \\
0.108-0.657\end{array}$ \\
\hline Biochemical & $\begin{array}{l}\text { Fasting blood glucose } \\
\text { HDL }\end{array}$ & $\begin{array}{c}0.056 \\
-\end{array}$ & $\begin{array}{c}1.288 \\
-\end{array}$ & $\begin{array}{c}0.994-1.670 \\
-\end{array}$ & $\begin{array}{l}0.103 \\
0.031 *\end{array}$ & $\begin{array}{l}1.302 \\
0.338\end{array}$ & $\begin{array}{l}0.948-1.786 \\
0.126-0.905\end{array}$ \\
\hline Physical fitness & $\begin{array}{l}\text { Chair sit-and-reach } \\
\text { Timed up and go } \\
\text { Back scratch }\end{array}$ & $\begin{array}{l}0.022^{*} \\
0.001 * * \\
0.002 * *\end{array}$ & $\begin{array}{l}1.032 \\
1.261 \\
1.038\end{array}$ & $\begin{array}{l}1.005-1.060 \\
1.097-1.450 \\
1.014-1.062\end{array}$ & $\begin{array}{c}0.020^{*} \\
0.016^{*} \\
<0.001 * * *\end{array}$ & $\begin{array}{l}1.039 \\
1.207 \\
1.054\end{array}$ & $\begin{array}{l}1.006-1.073 \\
1.036-1.407 \\
1.024-1.085\end{array}$ \\
\hline Cognitive function & $\begin{array}{l}\text { MMSE } \\
\text { MoCA } \\
\text { Digit symbol } \\
\text { Digit span } \\
\text { RAVLT T5 score }\end{array}$ & $\begin{array}{c}<0.00 I^{* * *} \\
0.003^{* *} \\
0.00 I^{* *} \\
0.047^{*} \\
<0.00 I^{* * *}\end{array}$ & $\begin{array}{l}0.846 \\
0.920 \\
0.853 \\
0.898 \\
0.949\end{array}$ & $\begin{array}{l}0.772-0.926 \\
0.87 I-0.972 \\
0.777-0.935 \\
0.807-0.998 \\
0.923-0.975\end{array}$ & $\begin{array}{c}0.001 * * \\
0.017^{*} \\
0.008^{* *} \\
0.039 * \\
<0.001 * * *\end{array}$ & $\begin{array}{l}0.829 \\
0.926 \\
0.869 \\
0.882 \\
0.935\end{array}$ & $\begin{array}{l}0.74 I-0.927 \\
0.869-0.986 \\
0.784-0.964 \\
0.782-0.994 \\
0.905-0.965\end{array}$ \\
\hline Psychosocial status & $\begin{array}{l}\text { WHODAS } 2.0 \\
\text { Flourishing Scale }\end{array}$ & $\begin{array}{l}0.009 * * \\
0.010 *\end{array}$ & $\begin{array}{l}1.126 \\
1.083\end{array}$ & $\begin{array}{l}1.030-1.231 \\
1.019-1.151\end{array}$ & $\begin{array}{c}0.099 \\
-\end{array}$ & $\begin{array}{c}1.073 \\
-\end{array}$ & $\begin{array}{c}0.987-1.167 \\
-\end{array}$ \\
\hline
\end{tabular}

Notes: $*_{p}<0.05 ; * * p<0.01 ; * * * p<0.001$.

Abbreviations: HDL, high-density lipoprotein; MMSE, Mini-Mental State Examination; MoCA, Montreal Cognitive Assessment; RAVLT, Rey Auditory Verbal Learning Test; WHODAS 2.0, World Health Organization Disability Assessment Schedule 2.0.

subjected to various factors such as the degree of $\mathrm{HL}$, demographic, socioeconomic and psychological factors. ${ }^{15}$ Besides, the prevalence of HFAHL (83.0\%) is slightly higher than PTAHL (75.5\%). Such observation is possible since ARHL, particularly the sensory presbycusis subtype, predominantly develops at higher frequency region first before deterioration to the lower frequency regions of the cochlea. $^{16}$
Our current findings showed that increasing age is a common risk factor for both PTAHL and HFAHL. The association between age and HL is well documented. It is predicted that starting from the second decade of life, the prevalence of HL will double for every ten years increase in age. ${ }^{1}$ Moreover, the prevalence of HL with a moderate or higher level of severity increases dramatically from $15.4 \%$ among those aged between 60 and 69 years old to 
Table 4 The Risk Factors Associated with PTAHL and HFAHL Among the Community-Dwelling Older Adults

\begin{tabular}{|l|l|c|c|c|c|c|c|}
\hline \multirow{2}{*}{ Risk Factors Category } & \multirow{2}{*}{ Item } & \multicolumn{3}{|c|}{ PTAHL } & \multicolumn{3}{c|}{ HFAHL } \\
\cline { 3 - 8 } & & p-value & Exp(B) & {$[95 \%$ CI] } & p-value & Exp(B) & {$[95 \%$ CI] } \\
\hline Sociodemographic & Age & $0.006^{* *}$ & 1.239 & $1.062-1.445$ & $0.043^{*}$ & 1.117 & $1.003-1.244$ \\
\hline Physical measurement & Waist circumference & $0.029^{*}$ & 1.158 & $1.015-1.322$ & - & - & - \\
Nutrition & Carbohydrate & - & - & - & $0.003^{* *}$ & 1.018 & $1.006-1.030$ \\
& Niacin & Potassium & $0.043^{*}$ & 0.909 & $0.831-0.988$ & - & - \\
$0.996-1.000$ & $<0.00 I^{* * *}$ & 0.998 & $0.997-0.999$ \\
\hline Cognitive function & RAVLT T5 score & $0.012^{*}$ & 0.905 & $0.838-0.978$ & $0.003^{* *}$ & 0.922 & $0.874-0.973$ \\
\hline
\end{tabular}

Notes: ${ }^{*} \mathrm{p}<0.05 ; * *_{\mathrm{p}}<0.01$; $* * * \mathrm{p}<0.001$.

Abbreviation: RAVLT, Rey Auditory Verbal Learning Test.

$58.2 \%$ among those over 90 years worldwide. ${ }^{2}$ The progression of HL with increasing age is mainly due to cochlear and neural degeneration that affects auditory processing and sound interpretation. ${ }^{17}$ Furthermore, the accumulated insults to the ear due to the constant exposure to various risk factors of HL may increase across the life span, contributing to higher risk of having HL as people live longer.

Our study demonstrated that higher waist circumference is associated with an increased risk of HL among older adults. There is evidence suggesting that waist circumference, a measurement of abdominal obesity and central adiposity is more prominent in predicting HL compared to BMI, which reflects the overall adiposity of the body. ${ }^{18,19}$ The effect of BMI on hearing levels is inconclusive. Adults with obesity were reported to be at a higher risk of having hearing impairments than their counterparts with normal BMI. ${ }^{20}$ This might be because of excessive adiposity can lead to obesity-induced oxidative stress, which may cause damage to the auditory structures of the inner ear. ${ }^{21}$ Besides, obesity and other associated comorbidities such as hypertension, cardiovascular diseases and diabetes mellitus can cause changes to the blood vessels and capillaries that supply the ear, disrupting the blood circulation in the inner ear and subsequently damaging the hair cells. ${ }^{20}$ In contrast, Kim et al found that underweight individuals are more likely to develop HL than those with normal BMI. ${ }^{22}$ These rather contradictory findings may be due to the deposition of fat around the abdominal has a stronger association with other obese-related comorbidities, which may contribute to the development and progression of $\mathrm{HL}$ as compared to overall adiposity. ${ }^{23}$

In this present study, we demonstrated that a higher carbohydrate intake is associated with an increased risk of having HL. Previously, Gopinath et al reported that high intake of glycemic load diet and total carbohydrate among older adults was associated with an increased risk of developing HL. ${ }^{24}$ Similarly, Rosenhall et al reported that high consumption of low molecular weight carbohydrates was correlated to poorer hearing thresholds at high frequencies. ${ }^{25}$ Besides, people with high consumption of carbohydrates, especially those simple carbohydrates and added sugars, are more likely to become obese and develop other comorbidities like cardiovascular diseases, dyslipidemia and diabetes mellitus. ${ }^{26}$ As discussed previously, obesity and comorbidities like cardiovascular diseases may affect the hearing by disrupting the blood flow in the inner ear. ${ }^{20}$

Contrary to our current findings, Spankovich et al demonstrated that a higher intake of carbohydrates confers protective effects against $\mathrm{HL}$ among older adults. ${ }^{27}$ This is possible since complex carbohydrates such as cereal fiber, whole grain and vegetables are food with low glycemic index and may lead to increased satiety and lower energy intake. $^{28}$ Previously, high consumption of food rich in complex carbohydrates and dietary fiber is associated with smaller waist circumference and reduced adiposity. ${ }^{29}$ Hence, different sources of carbohydrates may have discrete biological effects on the body and affect the hearing capacity differently.

Niacin, or better known as vitamin B3, is a precursor of the two biologically active coenzymes, namely the nicotinamide adenine dinucleotide (NAD) and nicotinamide adenine dinucleotide phosphate (NADP). ${ }^{30}$ Apart from its well-known physiological function in energy production, cellular signaling, DNA repair, and the central nervous system, the role of niacin in auditory function remains unclear. To date, there are only a few studies reporting 
the association of niacin intake with HL. Our findings showed that inadequate intake of niacin might increase the risk of having ARHL. Previously, Kang et al reported that niacin intake is correlated to better hearing acuity in only the univariate linear regression model, while Kim and Chung demonstrated that higher consumption of niacin reduced the risk of developing ARHL in older adults. $^{31,32}$ However, a recent study conducted by Choi et al showed that niacin intake was not associated with the development of ARHL. ${ }^{33}$ The possible mechanism underlying the protective effects of niacin against HL is that niacin can prevent the degeneration of the spiral ganglion neurons and preserve the synaptic contacts between the spiral ganglion neurons and hair cells via the activation of $\mathrm{NAD}^{+}$-SIRT3 pathway following intense exposure to noise, as demonstrated in vivo. ${ }^{34}$ Hence, further study is needed to confirm the role of niacin in the prevention of ARHL.

In agreement with our current findings, Jung et al demonstrated that higher potassium intake is associated with better hearing levels and lower prevalence of HL in adults. ${ }^{35}$ Potassium is one of the essential nutrients that help maintain fluids and electrolytes balance, the transmission of nerve impulse and muscle contraction in our body. ${ }^{36}$ A high potassium ion concentration is found in the cochlear endolymph and is required to generate endocochlear potential (EP) that drives the sensory transduction in the hair cells. ${ }^{37}$ During sound transduction, the vibration of the basilar membrane causes the opening of the specialized transduction channel on the stereocilia of hair cells. The potassium ions are driven into hair cells due to the presence of EP, hence exciting the hair cells. Disruption in potassium homeostasis in the endolymph due to degeneration of stria vascularis (a specialized ion transport structure in cochlear), reduced expression of specific potassium ion channels, and mutation in genes encoding the potassium-regulating proteins in the cochlear has been associated with sensorineural HL. ${ }^{38,39}$

However, despite playing an essential physiological role in sound transduction, the direct association between potassium intake and HL remains unclear. Previously, consumption of a high-potassium diet was demonstrated to increase the aldosterone level in the body and subsequently leads to the increased expression of $\mathrm{Na}^{+} /-\mathrm{K}^{+}$ ATPase and NKCC1 in the stria vascularis, suggesting that higher intake of potassium could help in the maintenance of EP in the endolymph and protect against
ARHL. ${ }^{40-43}$ Furthermore, a high potassium diet may also protect against HL indirectly via its beneficial effects on hypertension and glycemic control. ${ }^{36}$

Current findings also demonstrated that older adults with HL had lower scores in the RAVLT cognitive test. The RAVLT is a neuropsychological tool widely used to assess functions such as attention, memory, and learning ability in the auditory-verbal domain. ${ }^{44}$ Although hearing impairment is widely accepted as one of the causes of cognitive impairment among older adults, this association may be directional because HL and cognitive impairment share the same common risk factors and pathogenesis mechanisms, such as cardiovascular diseases, microcirculation disorders, oxidative stress and inflammation. ${ }^{45,46}$ However, the auditory-verbal memory of participants with hearing impairment should be analyzed carefully to avoid any misleading interpretation. It is suggested that the participants' hearing capacity may affect the RAVLT test outcomes since the administration of RAVLT requires communication between the assessor and participant and the test items are presented verbally to the participants. ${ }^{47}$ Cognitively intact adults with HL performed significantly worse than their normal-hearing counterparts in auditoryverbal memory tests, thus underestimating the actual cognitive performance of the participants diagnosed with HL. ${ }^{48}$

In the present study, participants with HL generally had lower performance in other cognitive tests as well. However, only RAVLT T5 scores appeared to be associated with HL in the final multiple logistic regression model. Hence, instead of being recognized as cognitive impaired, we cannot rule out the possibility of lower performance in the RAVLT test among the participants with HL was due to their inability to listen correctly to the commands and test items presented verbally by the assessor. Furthermore, a recent study conducted by Füllgrabe (2020) demonstrated that despite with perfect audibility of the test items, the cognitive test performance of the participants was compromised in the simulated-HL condition. $^{49}$ This is possibly due to the additional cognitive resources required for auditory perceptual processing following simulated-HL condition, limiting the remaining cognitive resources for the execution of other cognitive processes. ${ }^{46}$ Future studies involving a prospective cohort population are needed to verify the relationship between the RAVLT scores and HL.

As there is no cure for HL, the hearing aid is one of the options to reduce the detrimental effects of HL. However, 
in Malaysia, the rate of hearing aid adoption was only $2.7 \%$ among older adults with HL. ${ }^{50}$ Thus, the determination of modifiable risk factors of HL as presented in the present study may help audiologists or policymakers in designing a more comprehensive and effective hearing health awareness or HL prevention program.

\section{Conclusion}

In conclusion, increasing age, having higher waist circumference, lower intake of niacin and potassium, higher intake of carbohydrates and lower RAVLT T5 score were associated with increased risk of ARHL. Identifying these risk factors may help develop preventive and management strategies for ARHL in older adults. Nevertheless, this study has demonstrated its strength in using objective measures to assess HL and its association using a wide range of health factors. However, due to the crosssectional nature of this study, which makes the causal link between the risk factors and ARHL inconclusive, a future longitudinal-cohort study is needed.

\section{Abbreviations}

ADL, Activities of Daily Living; ARHL, age-related hearing loss; BMI, body mass index; EP, endocochlear potential; HDL, high-density lipoprotein; HFA, highfrequency pure-tone average; HFAHL, hearing loss based on high-frequency pure-tone average; HL, hearing loss; IADL, Instrumental Activity of Daily Living; LDL, low-density lipoprotein; LRGS-TUA, Long-term Research Grant Scheme - Towards Useful Ageing; MMSE, Mini-Mental State Examination; MoCA, Montreal Cognitive Assessment; MOSS, Medical Outcome Social Support; NAD, nicotinamide adenine dinucleotide; NADP, nicotinamide adenine dinucleotide phosphate; NHMS, National Health Morbidity Survey; PTA, pure-tone average; PTAHL, hearing loss based on pure-tone average; RAVLT, Rey Auditory Verbal Learning Test; WHODAS 2.0, World Health Organization Disability Assessment Schedule 2.0; YLDs, years lived with disability.

\section{Data Sharing Statement}

The data that support the findings of this study are available from the corresponding author upon reasonable request.

\section{Acknowledgments}

The authors thank the participants and the team members of the study for their participation and support given.

\section{Author Contributions}

All authors made a significant contribution to the work reported, whether that is in the conception, study design, execution, acquisition of data, analysis and interpretation, or in all these areas; took part in drafting, revising or critically reviewing the article; gave final approval of the version to be published; have agreed on the journal to which the article has been submitted; and agree to be accountable for all aspects of the work.

\section{Funding}

This work was supported by the Ministry of Higher Education Malaysia under the Long-term Research Grant Scheme (LRGS/1/2019/UM-UKM/1/4 and LRGS/BU/ 2012/UKM-UKM/K/01).

\section{Disclosure}

The authors report no conflicts of interest in this work.

\section{References}

1. Bowl MR, Dawson SJ. Age-related hearing loss. Cold Spring Harb Perspect Med. 2019;9(8):1-15. doi:10.1101/cshperspect.a033217

2. World Health Organization. World Report on Hearing. World Health Organization; 2021.

3. Haile LM, Kamenov K, Briant PS, et al. Hearing loss prevalence and years lived with disability, 1990-2019: findings from the Global Burden of Disease Study 2019. Lancet. 2021;397(10278):996-1009. doi:10.1016/S0140-6736(21)00516-X

4. Walling AD, Dickson GM. Hearing loss in older adults. Am Fam Physician. 2012;85(12):1150-1156. doi:10.1001/jama.2012.321

5. Weinstein BE. The cost of age related hearing loss: to treat or not to treat? Speech, Lang Hear. 2019;22(1):9-15. doi:10.1080/ 2050571X.2018.1533622

6. Ministry of Health Malaysia. Chapter 2 - Population Health. Malaysia's Health Report 2007; 2007. 155-166. Available form: http://www2.moh.gov.my/images/gallery/publications/mh/ MalaysiaHealth2007-2.pdf. Accessed November 24, 2021.

7. Shahar S, Omar A, Vanoh D, et al. Approaches in methodology for population-based longitudinal study on neuroprotective model for healthy longevity (TUA) among Malaysian Older Adults. Aging Clin Exp Res. 2016;28(6):1089-1104. doi:10.1007/s40520-015-0511-4

8. Trzepacz PT, Hochstetler H, Wang S, Walker B, Saykin AJ. Relationship between the Montreal Cognitive Assessment and Mini-mental State Examination for assessment of mild cognitive impairment in older adults. BMC Geriatr. 2015;15(1):1-9. doi:10.1186/s12877-015-0103-3

9. Mukari SZMS, Ishak WS, Maamor N, Wan Hashim WF, Preliminary Study A. Investigating the Association between Hearing Acuity and a Screening Cognitive Tool. Ann Otol Rhinol Laryngol. 2017;126 (10):697-705. doi:10.1177/0003489417727547

10. Mukari SZMS, Maamor N, Ishak WS, Hashim WFW. Hearing Loss and Risk Factors among Community Dwelling Older Adults in Selangor. Sains Malaysiana. 2016;45(9):1405-1411.

11. Mah HY, Ishak WS, Abd Rahman MH. Prevalence and risk factors of dual sensory impairment among community-dwelling older adults in Selangor: a secondary data analysis. Geriatr Gerontol Int. 2020;20 (10):911-916. doi:10.1111/ggi.14011 
12. Harith AA, Ahmad NA, Sahril N, et al. Prevalence and determinants of hearing disability among older persons in Malaysia: finding of National Health Morbidity Survey (NHMS) 2018. Geriatr Gerontol Int. 2020;20 Suppl 2:43-48. doi:10.1111/ggi.14039

13. Choi JE, Moon IJ, Baek SY, Kim SW, Cho YS. Discrepancies between self-reported hearing difficulty and hearing loss diagnosed by audiometry: prevalence and associated factors in a national survey. BMJ Open. 2019;9(4):1-17. doi:10.1136/bmjopen-2018022440

14. Ishak WS, Mukari SZMS, Maamor N, Hashim WFW. Validation of self-reported hearing loss among multi-ethnic community dwelling older adults in Malaysia. J Clin Diagnostic Res. 2017;11(10):MC01MC05. doi:10.7860/JCDR/2017/28144.10756

15. Kim SY, Kim HJ, Kim MS, Park B, Kim JH, Choi HG. Discrepancy between self-assessed hearing status and measured audiometric evaluation. PLoS One. 2017;12(8):1-14. doi:10.1371/journal. pone.0182718

16. Wu PZ, O’Malley JT, de Gruttola V, Charles Liberman M. Agerelated hearing loss is dominated by damage to inner ear sensory cells, not the cellular battery that powers them. J Neurosci. 2020;40 (33):6357-6366. doi:10.1523/JNEUROSCI.093720.2020

17. Tu NC, Friedman RA. Age-related hearing loss: unraveling the pieces. Laryngoscope Investig Otolaryngol. 2018;3(2):68-72. doi:10.1002/lio2.134

18. Curhan SG, Eavey R, Wang M, Stampfer MJ, Curhan GC. Body mass index, waist circumference, physical activity, and risk of hearing loss in women. Am J Med. 2013;126(12):1142.e1-1142.e8. doi:10.1016/j.amjmed.2013.04.026

19. Yang JR, Hidayat K, Chen CL, et al. Body mass index, waist circumference, and risk of hearing loss: a meta-analysis and systematic review of observational study. Environ Health Prev Med. 2020;25 (1):1-9. doi:10.1186/s12199-020-00862-9

20. Dhanda N, Taheri S. A narrative review of obesity and hearing loss. Int J Obes. 2017;41(7):1066-1073. doi:10.1038/ijo.2017.32

21. Poirrier A, Pincemail J, Van Den Ackerveken P, P. Lefebvre P, Malgrange B. Oxidative Stress in the Cochlea: an Update. Curr Med Chem. 2010;17(30):3591-3604. doi:10.2174/092986710792 927895

22. Kim SH, Won YS, Kim MG, Baek YJ, Oh IH, Yeo SG. Relationship between obesity and hearing loss. Acta Otolaryngol. 2016;136 (10):1046-1050. doi:10.1080/00016489.2016.1179787

23. Lee MJ, Wu Y, Fried SK. Adipose tissue heterogeneity: implication of depot differences in adipose tissue for obesity complications. Mol Aspects Med. 2013;34(1):1-11. doi:10.1016/j. mam.2012.10.001

24. Gopinath B, Flood VM, McMahon CM, Burlutsky G, Brand-Miller J, Mitchell P. Dietary glycemic load is a predictor of age-related hearing loss in older adults. $J$ Nutr. 2010;140(12):2207-2212. doi:10.3945/ jn.110.128462

25. Rosenhall U, Idrizbegovic E, Hederstierna C, Rothenberg E. Dietary habits and hearing. Int J Audiol. 2015;54(September 2014):S53-S56. doi:10.3109/14992027.2014.972524

26. Ludwig DS, Ebbeling CB. The carbohydrate-insulin model of obesity: beyond "calories in, calories out.". JAMA Intern Med. 2018;178 (8):1098-1103. doi:10.1001/jamainternmed.2018.2933

27. Spankovich C, Hood LJ, Silver HJ, Lambert W, Flood VM, Mitchell P. Associations between diet and both high and low pure tone averages and transient evoked otoacoustic emissions in an older adult population-based study. J Am Acad Audiol. 2011;22(1):49-58. doi:10.3766/jaaa.22.1.6

28. Batista SM, Moreira EAM, Fiates GMR, de Assis MAA, Teixeira E. Effect of low Glycaemic Index Diets on Satiety. Br Food J. 2014;116 (8):1233-1246. doi:10.1108/BFJ-08-2012-0208

29. Lofley AC, Root MM. Macronutrients Association with Change in Waist and Hip Circumference Over 9 Years. J Am Coll Nutr. 2017;36 (1):57-63. doi:10.1080/07315724.2016.1183241
30. Gasperi V, Sibilano M, Savini I, Catani MV. Niacin in the central nervous system: an update of biological aspects and clinical applications. Int J Mol Sci. 2019;20(4):1-26. doi:10.3390/ ijms20040974

31. Kang JW, Choi HS, Kim K, Choi JY. Dietary vitamin intake correlates with hearing thresholds in the older population: the Korean National Health and Nutrition Examination Survey. Am J Clin Nutr. 2014;99(6):1407-1413. doi:10.3945/ajcn.113.072793

32. Kim TS, Chung JW. Associations of dietary riboflavin, niacin, and retinol with age-related hearing loss: an analysis of Korean national health and nutrition examination survey data. Nutrients. 2019;11:4. doi:10.3390/nu11040896

33. Choi JE, Ahn J, Moon IJ. Associations between age-related hearing loss and dietary assessment using data from Korean national health and nutrition examination survey. Nutrients. 2021;13:4. doi:10.3390/ nu13041230

34. Brown KD, Maqsood S, Huang JY, et al. Activation of SIRT3 by the $\mathrm{NAD}^{+}$precursor nicotinamide riboside protects from noise-induced hearing loss. Cell Metab. 2014;20(6):1059-1068. doi:10.1016/j. cmet.2014.11.003

35. Jung DJ, Lee JY, Cho KH, Lee KY, Do JY, Kang SH. Association between a High-Potassium Diet and Hearing Thresholds in the Korean Adult Population. Sci Rep. 2019;9(1):1-11. doi:10.1038/ s41598-019-45930-5

36. Stone MS, Martyn L, Weaver CM. Potassium intake, bioavailability, hypertension, and glucose control. Nutrients. 2016;8(7):1-13. doi: $10.3390 /$ nu8070444

37. Marcus DC, Wangemann P. Cochlear and Vestibular Function and Dysfunction. Elsevier Inc; 2010; doi:10.1016/B978-0-12-3743732.00021-2

38. Peixoto Pinheiro B, Vona B, Löwenheim H, Rüttiger L, Knipper M, Adel Y. Age-related hearing loss pertaining to potassium ion channels in the cochlea and auditory pathway. Pflugers Arch Eur J Physiol. 2021;473(5):823-840. doi:10.1007/s00424-020-02496-w

39. Locher H, de Groot JCMJ, van Iperen L, Huisman MA, Frijns JHM. Development of the stria vascularis and potassium regulation in the human fetal cochlea: insights into hereditary sensorineural hearing loss. Dev Neurobiol. 2015;75(11):1219-1240. doi:10.1002/ dneu. 22279

40. Cate WJF, Curtis LM, Rarey KE. Effects of low-sodium, high-potassium dietary intake on cochlear lateral wall $\mathrm{Na}+, \mathrm{K}(+)--$ ATPase. Eur Arch Oto-Rhino-Laryngology. 1994;251(1):6-11. doi:10.1007/BF00175950

41. Ding B, Frisina RD, Zhu X, Sakai Y, Sokolowski B, Walton JP. Direct control of $\mathrm{Na}+-\mathrm{K}+-2 \mathrm{Cl}-$ cotransport protein $(\mathrm{NKCC} 1)$ expression with aldosterone. Am J Physiol. 2014;306(1):66-75. doi:10.1152/ajpcell.00096.2013

42. Tadros SF, Frisina ST, Mapes F, Frisina DR, Frisina RD. Higher serum aldosterone correlates with lower hearing thresholds: a possible protective hormone against presbycusis. Hear Res. 2005;209(1-2):10-18. doi:10.1016/j.heares.2005.05.009

43. Halonen J, Hinton AS, Frisina RD, Ding B, Zhu X, Walton JP. Longterm treatment with aldosterone slows the progression of age-related hearing loss. Hear Res. 2016;336:63-71. doi:10.1016/j. heares.2016.05.001

44. Strauss E, Sherman EMS, Spreen O. A Compendium of Neuropsychological Tests: Administration, Norms, and Commentary. 3rd ed. Oxford University Press; 2006.

45. Fortunato S, Forli F, Guglielmi V, et al. A review of new insights on the association between hearing loss and cognitive decline in ageing. Acta Otorhinolaryngol Ital. 2016;36(3):155-166. doi:10.14639/0392100X-993

46. Uchida Y, Sugiura S, Nishita Y, Saji N, Sone M, Ueda H. Age-related hearing loss and cognitive decline - the potential mechanisms linking the two. Auris Nasus Larynx. 2019;46(1):1-9. doi:10.1016/j. anl.2018.08.010 
47. van Boxtel MP, van Beijsterveldt CE, Houx PJ, Anteunis LJ, Metsemakers JF, Jolles J. Mild hearing impairment can reduce verbal memory performance in a healthy adult population. J Clin Exp Neuropsychol. 2000;22(1):147-154. doi:10.1076/1380-3395(200002) 22:1;1-8;FT147

48. Wong CG, Rapport LJ, Billings BA, Ramachandran V, Stach BA. Hearing loss and verbal memory assessment among older adults. Neuropsychology. 2019;33(1):47-59. doi:10.1037/neu0000489
49. Füllgrabe C. On the Possible Overestimation of Cognitive Decline: the Impact of Age-Related Hearing Loss on Cognitive-Test Performance. Front Neurosci. 2020;14(June). doi:10.3389/ fnins.2020.00454

50. Mukari SZMS, Wan hashim WF. Self-Perceived Hearing Loss, Hearing-Help Seeking and Hearing Aid Adoption Among Older Adults in Malaysia. Ann Otol Rhinol Laryngol. 2018;127 (11):798-805. doi:10.1177/0003489418795982

\section{Publish your work in this journal}

Clinical Interventions in Aging is an international, peer-reviewed journal focusing on evidence-based reports on the value or lack thereof of treatments intended to prevent or delay the onset of maladaptive correlates of aging in human beings. This journal is indexed on PubMed Central, MedLine, CAS, Scopus and the Elsevier
Bibliographic databases. The manuscript management system is completely online and includes a very quick and fair peer-review system, which is all easy to use. Visit http://www.dovepress.com/ testimonials.php to read real quotes from published authors. 